\title{
Supporting Information for: Van der Waals Potential Energy Surfaces from the Exchange-Hole Dipole Moment Dispersion Model
}

TABLE A.1. Optimized bond lengths of linear molecules with respect to the center of mass as the origin in $\AA$ with dispersioncorrected functionals.

\begin{tabular}{c|c|ccc}
\hline Molecule & Bond & PW86PBE & PW1PBE & HFPBE \\
\hline $\mathrm{CO}$ & $\mathrm{C}-\mathrm{X}$ & 0.6488 & 0.6434 & 0.6248 \\
& $\mathrm{O}-\mathrm{X}$ & 0.4866 & 0.4826 & 0.4686 \\
\hline $\mathrm{H}_{2}$ & $\mathrm{H}-\mathrm{X}$ & 0.3731 & 0.3715 & 0.3653 \\
\hline $\mathrm{N}_{2}$ & $\mathrm{~N}-\mathrm{X}$ & 0.5511 & 0.5463 & 0.5291 \\
\hline $\mathrm{HF}$ & $\mathrm{H}-\mathrm{X}$ & 0.8385 & 0.8302 & 0.7997 \\
& $\mathrm{~F}-\mathrm{X}$ & 0.0932 & 0.0922 & 0.0889 \\
\hline $\mathrm{HCl}$ & $\mathrm{H}-\mathrm{X}$ & 1.2177 & 1.2098 & 1.1839 \\
& $\mathrm{Cl}-\mathrm{X}$ & 0.0716 & 0.0712 & 0.0696 \\
\hline $\mathrm{HBr}$ & $\mathrm{H}-\mathrm{X}$ & 1.3953 & 1.3861 & 1.3539 \\
& $\mathrm{Br}-\mathrm{X}$ & 0.0399 & 0.0396 & 0.0387 \\
\hline $\mathrm{CO}_{2}$ & $\mathrm{C}-\mathrm{O}$ & 1.1717 & 1.1609 & 1.1246 \\
\hline $\mathrm{OCS}$ & $\mathrm{O}-\mathrm{X}$ & 1.6955 & 1.6825 & 1.6389 \\
& $\mathrm{C}-\mathrm{X}$ & 0.5267 & 0.5256 & 0.5241 \\
& $\mathrm{~S}-\mathrm{X}$ & 1.0452 & 1.0383 & 1.0160 \\
\hline $\mathrm{HCN}^{\mathrm{H}}$ & $\mathrm{H}-\mathrm{X}$ & 1.5748 & 1.5657 & 1.5341 \\
& $\mathrm{C}-\mathrm{X}$ & 0.5021 & 0.4978 & 0.4826 \\
& $\mathrm{~N}-\mathrm{X}$ & 0.6553 & 0.6504 & 0.6328 \\
\hline $\mathrm{C}_{2} \mathrm{H}_{2}$ & $\mathrm{C}-\mathrm{X}$ & 0.6030 & 0.5992 & 0.5853 \\
& $\mathrm{H}-\mathrm{X}$ & 1.6713 & 1.6631 & 1.6338 \\
\hline
\end{tabular}

Potential energy surfaces for selected vdW complexes: note that the units of the Jacobi coordinate, $R$, corresponding to the intermolecular distance are shown as either Ångstrom $(\AA)$ or Bohr $\left(a_{0}\right)$ to facilitate comparison with reference literature PES. The contours of all PES are in terms of wavenumbers $\left(\mathrm{cm}^{-1}\right)$ unless otherwise stated.

FIG. A.1. PES of Rg-H $\mathrm{H}_{2}$ with XDM-corrected functionals for Ne (top) and Kr (bottom). The distance $R$ is given in units of Bohr for Ne and Ångstrom for Kr to facilitate comparison with the reference PES, available in Ref. 1 for Ne and Ref. 2 for Kr.
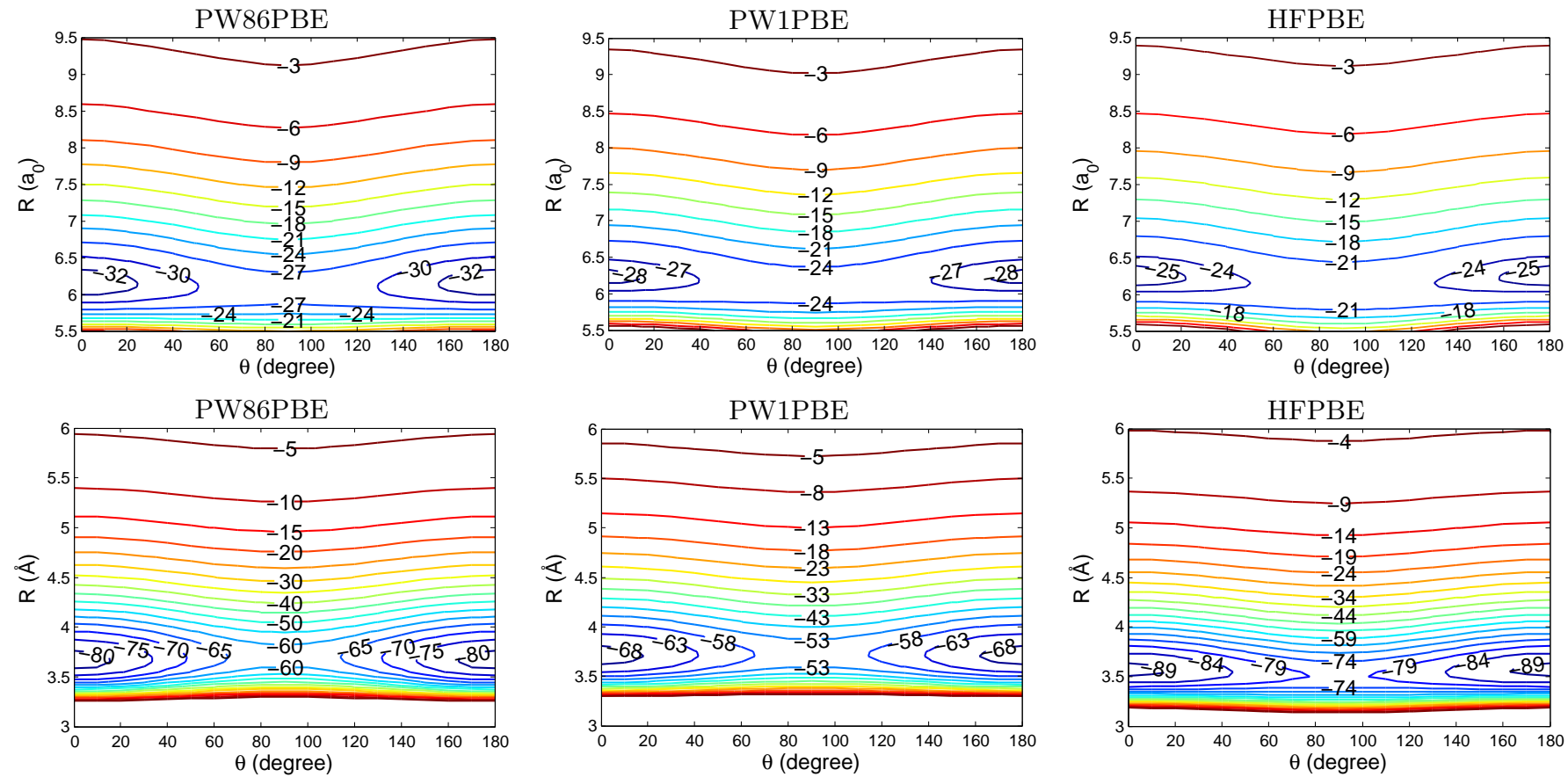
FIG. A.2. PES of Rg- $\mathrm{N}_{2}$ with XDM-corrected functionals for Ne (top), Ar (center), and Kr (bottom). The distance $R$ is given in units of Ångstrom for $\mathrm{Ne}$ and $\mathrm{Ar}$ and in units of Bohr for Kr to facilitate comparison with the reference PES, available in Refs. 3, 4, and 5 for $\mathrm{Ne}$, Ar, and Kr, respectively.
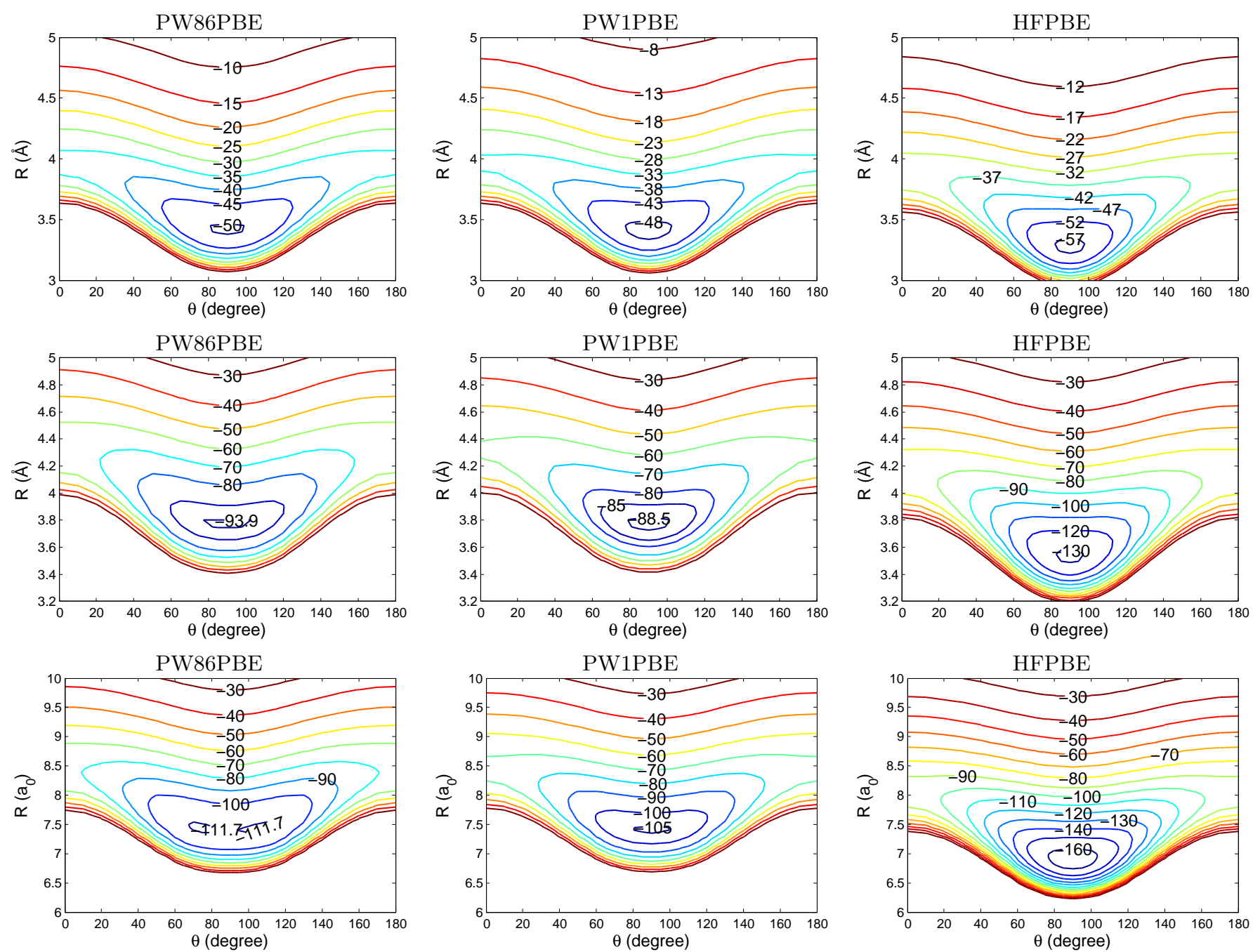
FIG. A.3. PES of Rg-CO with XDM-corrected functionals, for He, Ne, Ar, and Kr (top to bottom). The distance $R$ is given in units of Bohr for He, Ne, and Kr and in units of Ångstrom for Ar to facilitate comparison with the reference PES, available in Refs. 6, 7, 8, and 9 for $\mathrm{He}, \mathrm{Ne}, \mathrm{Ar}$, and $\mathrm{Kr}$, respectively.
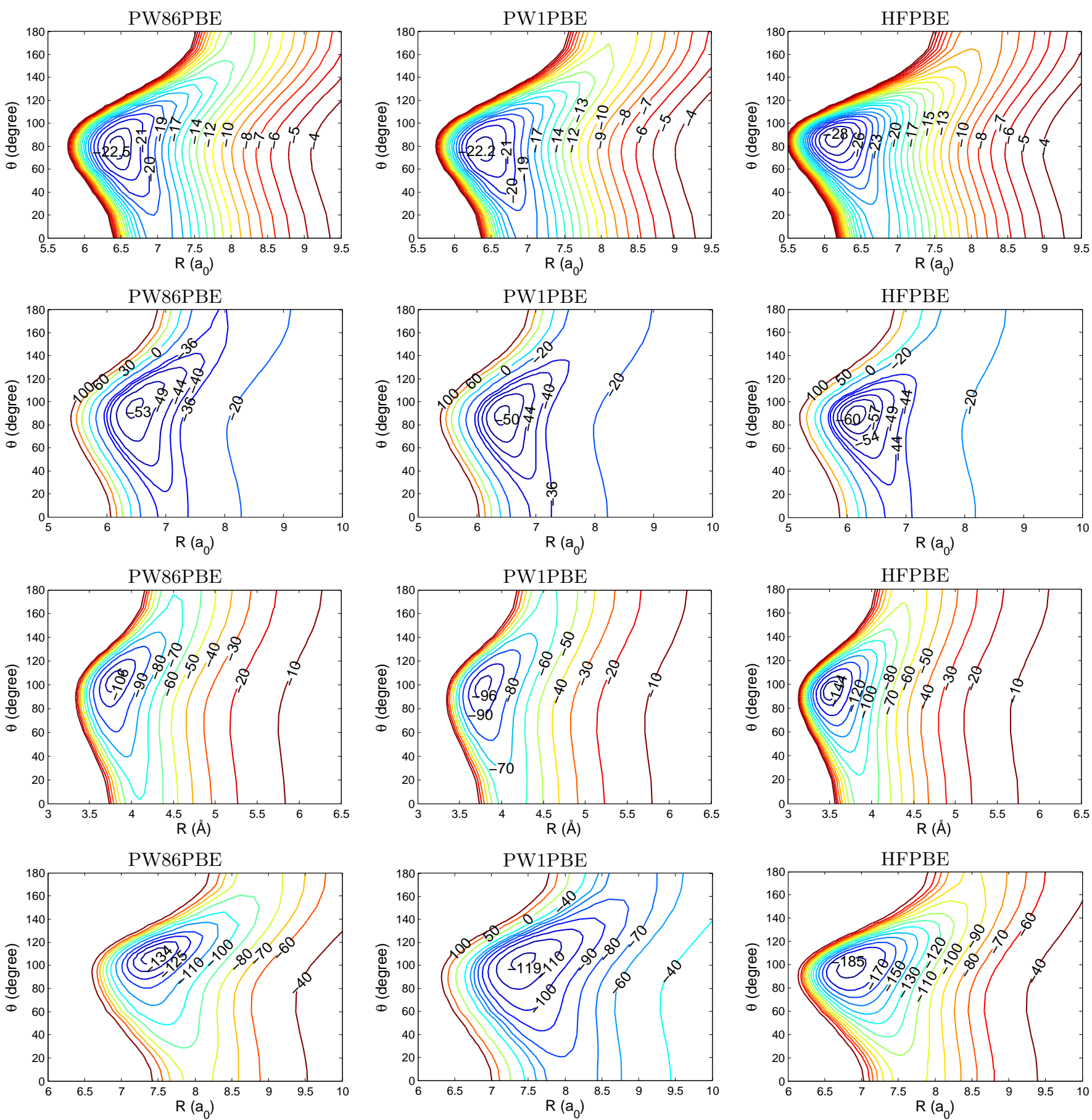
FIG. A.4. PES of Rg-HF with XDM-corrected functionals for He (top) and Kr (bottom). The distance $R$ is given in units of Angstrom for He and in units of Bohr for $\mathrm{Kr}$ to facilitate comparison with the reference PES, available in Ref. 10 for He and in Ref. 11 for Kr.
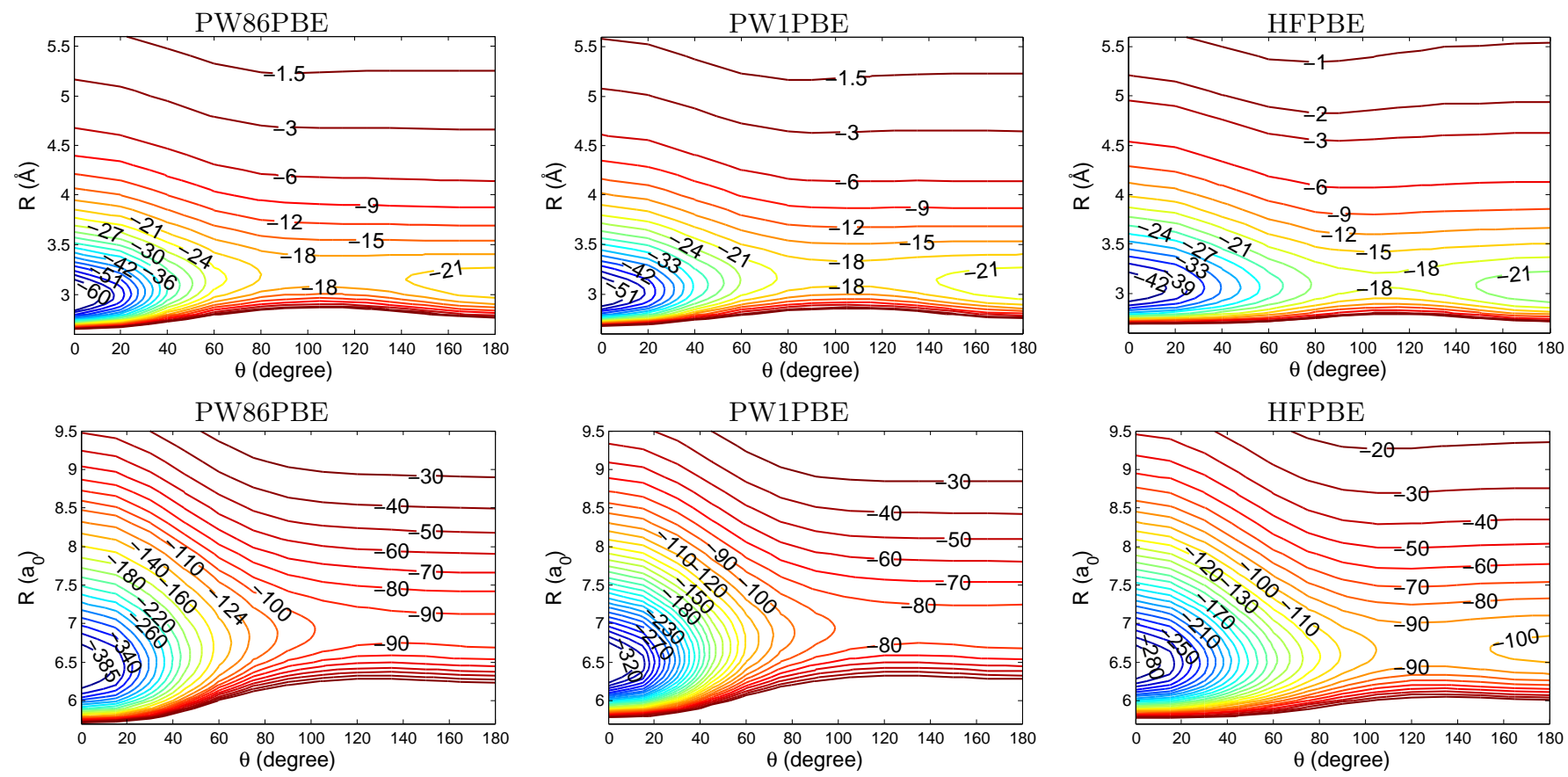

FIG. A.5. PES of Rg- $\mathrm{HCl}$ with XDM-corrected functionals for He (top) and Ne (bottom). The distance $R$ is given in units of Ångstrom. Reference PES are available in Ref. 12 for He and in Ref. 13 for Ne.
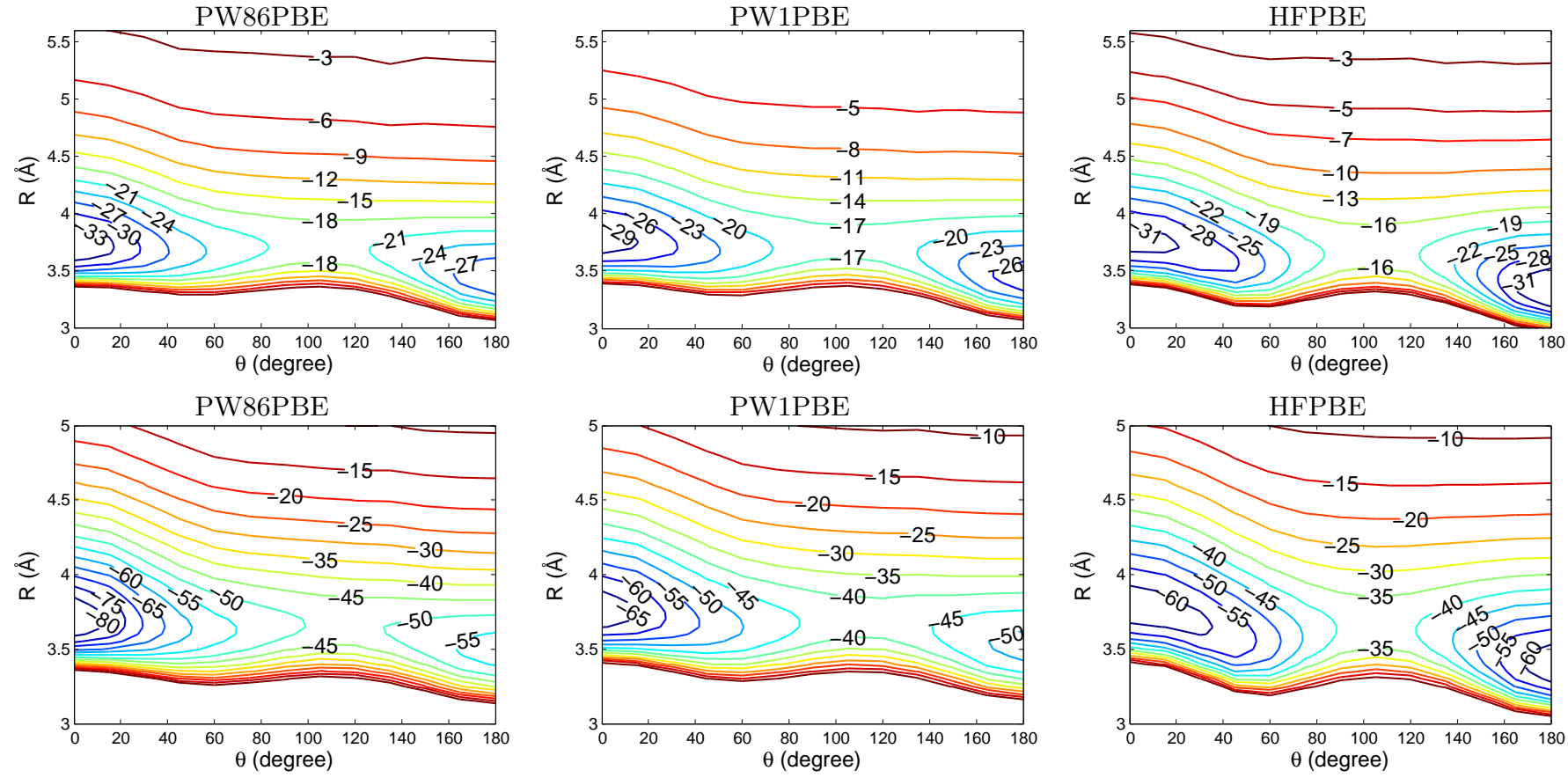
FIG. A.6. PES of Ne-HBr with XDM-corrected functionals. The distance $R$ is given in units of Ångstrom. The reference PES is available in Ref. 14 .
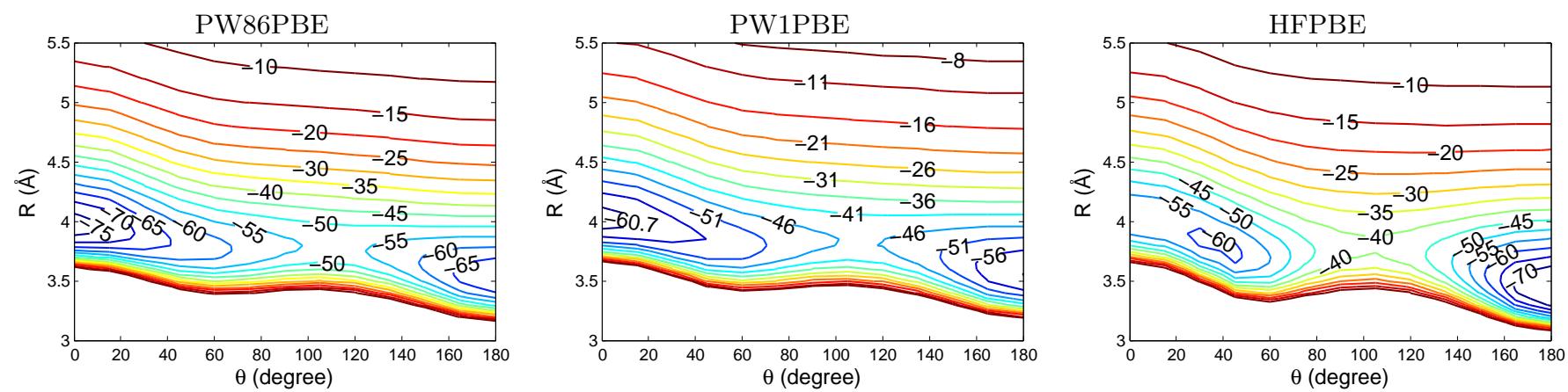

FIG. A.7. PES of Rg- $\mathrm{CO}_{2}$ with XDM-corrected functionals for He (top), Ne (center), and Kr (bottom). The distance $R$ is given in units of Bohr. Reference PES are available in Refs. 15, 16, and 17 for He, Ne, and Kr, respectively.
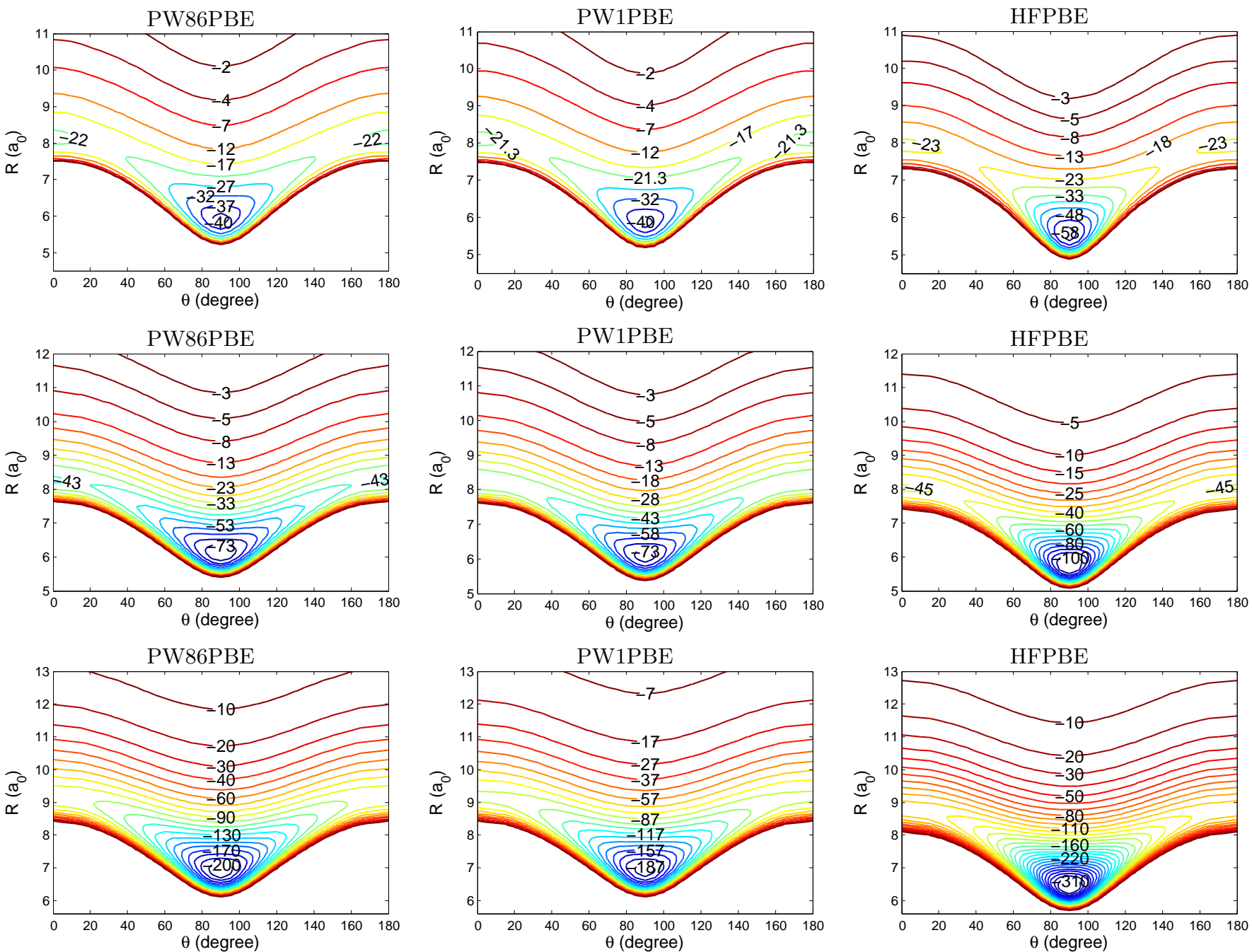
FIG. A.8. PES of Rg-OCS with XDM-corrected functionals for Ne (top), Ar (center), and Kr (bottom). The distance $R$ is given in units of Ångstrom for Ne and Ar and in units of Bohr for Kr to facilitate comparison with the reference PES, available in Refs. 18, 19, and 20 for $\mathrm{Ne}, \mathrm{Ar}$, and $\mathrm{Kr}$, respectively.
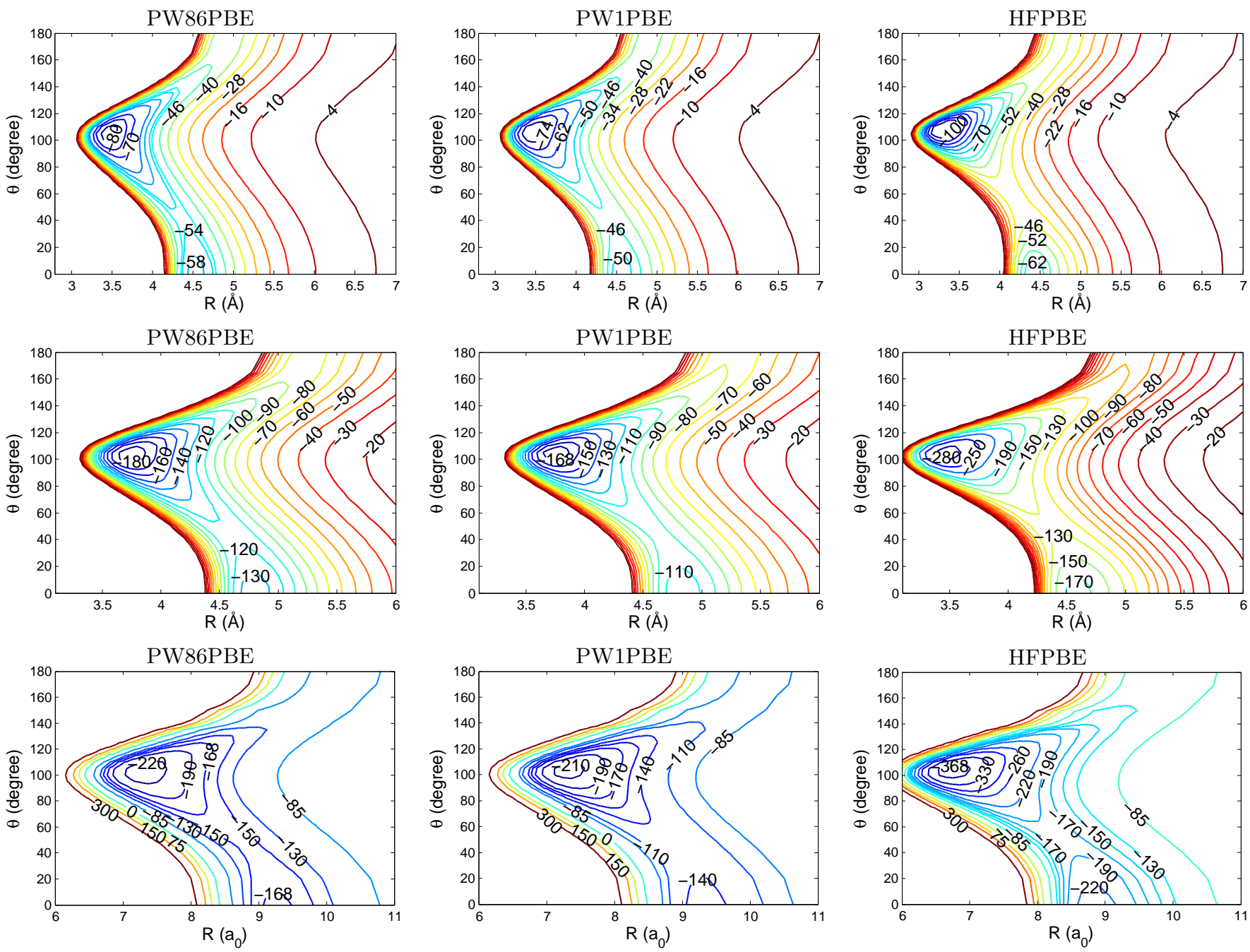
FIG. A.9. PES of Rg-HCN with XDM-corrected functionals for He, Ne, Ar, and Kr (top to bottom). The contours of the PES are in terms of miliHartrees $\left(\mathrm{mE}_{\mathrm{h}}\right)$. The distance $R$ is given in units of Bohr. All reference PES are available in Ref. 21.
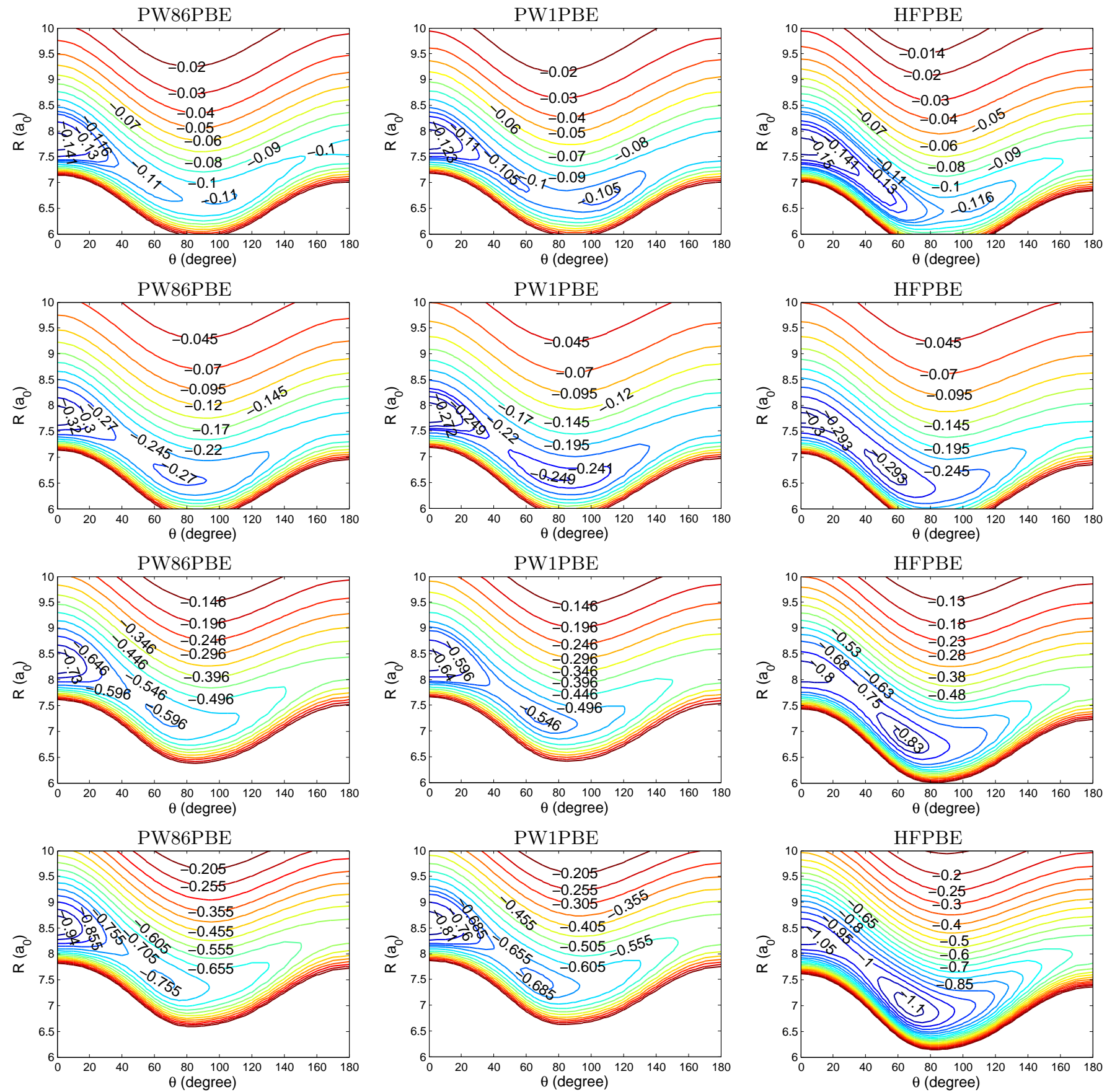
FIG. A.10. PES of $\mathrm{Rg}-\mathrm{C}_{2} \mathrm{H}_{2}$ with XDM-corrected functionals for He, Ne, Ar, and $\mathrm{Kr}$ (top to bottom). The distance $R$ is given in units of Ångstrom. Reference PES are available in Ref. 22 for He, Ne, and Ar, and in Ref. 23 for Kr.
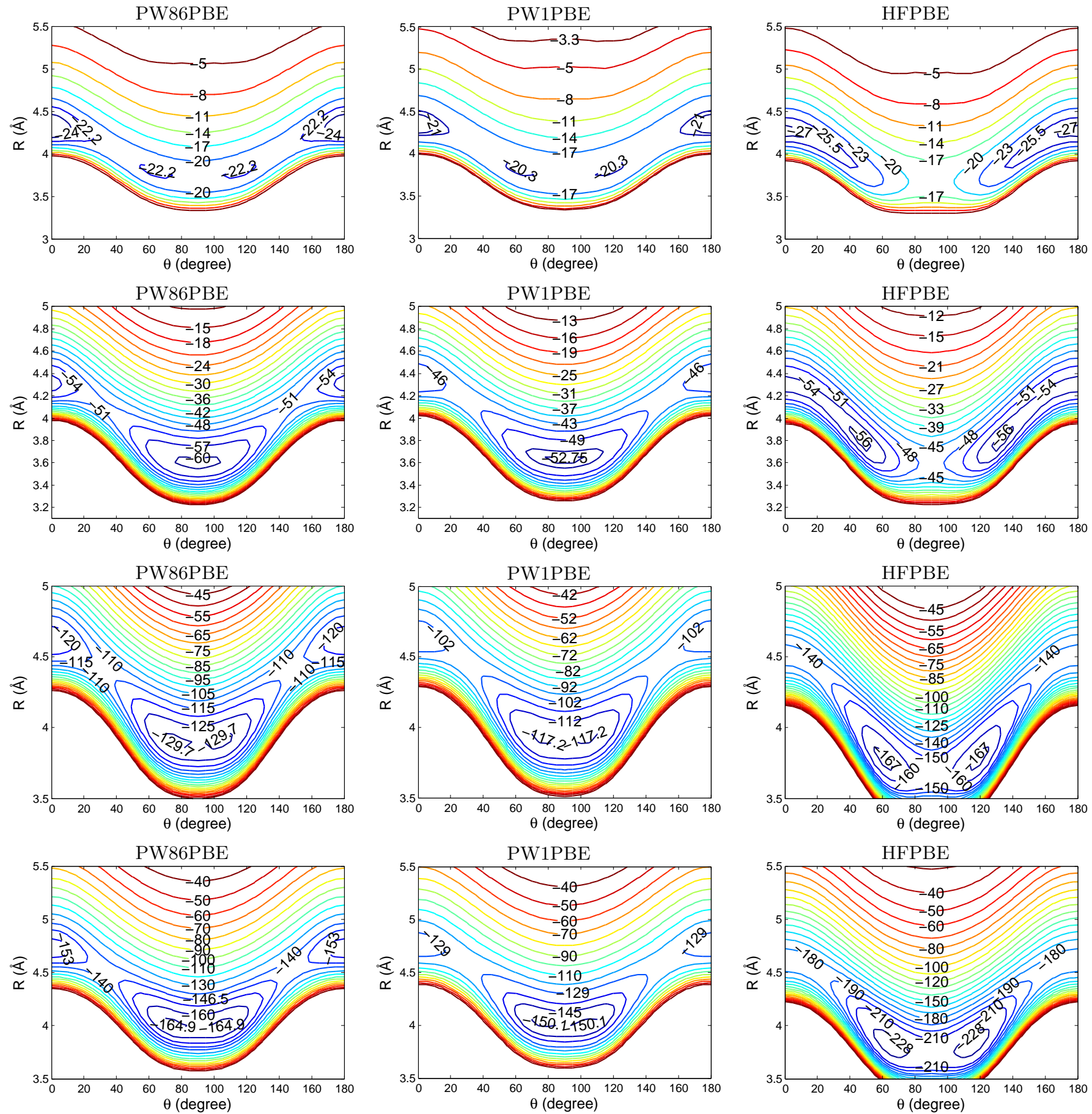

${ }^{1}$ F. Lique, A new ab initio potential energy surface for the NeH2 interaction, Chem. Phys. Lett. 471, $54-58$ (2009).

2 Y. Zhou and D. Xie, Three-dimensional ab initio potential-energy surface and rovibrational spectra of the H2Kr complex, J. Chem. Phys. 123, 134323 (2005).

${ }^{3}$ C. R. Munteanu, J. L. Cacheiro, and B. Fernández, Accurate intermolecular ground state potential of the NeN2 van der Waals complex, J. Chem. Phys. 120, 9104-9112 (2004).

${ }^{4}$ C. R. Munteanu, J. L. Cacheiro, and B. Fernández, Accurate intermolecular ground state potential of the Ar-N2 van der 
Waals complex, J. Chem. Phys. 121, 10419-10425 (2004).

${ }^{5}$ C. Zhang, Z. Wang, and E. Feng, Three-dimension potential energy surface and rovibrational spectra of the KrN2 complex, Chem. Phys. Lett. 517, $16-21$ (2011).

6 K. Peterson and G. McBane, A hierarchical family of three-dimensional potential energy surfaces for He-CO, J. Chem. Phys. 123, 084314 (2005).

7 Z. Wang, E. Feng, H. Yu, C. Zhang, and J. Du, A new ab initio potential energy surface for the NeCO complex with the vibrational coordinate dependence, J. Chem. Phys. 134, 024320 (2011).

8 R. R. Toczyłowski and S. M. Cybulski, An ab initio study of the potential energy surface and spectrum of ArCO, J. Chem. Phys. 112, 4604-4612 (2000).

9 E. Feng, Z. Wang, M. Gong, and Z. Cui, Interaction of CO with Kr: Potential energy surface and bound states, J. Chem. Phys. 127, 174301 (2007).

10 J. L. Cagide Fajín, B. Fernández, A. Mikosz, and D. Farrelly, Accurate computations of the rovibrational spectrum of the HeHF van der Waals complex, Mol. Phys 104, 1413-1420 (2006).

11 Z. Wang, C. Zhang, E. Feng, H. Yu, and J. Du, The rovibrational structure of the KrHF complex from an ab initio interaction potential, Chem. Phys. Lett. 501, $206-210$ (2011).

12 J. L. Cagide Fajín and B. Fernández, Accurate intermolecular ground state potential of the HeHCl van der Waals complex, Chem. Phys. Lett. 419, 55 - 58 (2006).

13 J. L. Cagide Fajín, J. Lopez Cacheiro, and B. Fernández, Accurate intermolecular ground state potential of the Ne-HCl van der Waals complex, J. Chem. Phys. 121, 4599-4604 (2004).

14 P. Slavíček, M. Roeselová, P. Jungwirth, and B. Schmidt, Preference of cluster isomers as a result of quantum delocalization: Potential energy surfaces and intermolecular vibrational states of NeHBr, NeHI, and HI(Ar)n(n=16), J. Chem. Phys. 114, 1539-1548 (2001).

15 H. Ran and D. Xie, A new potential energy surface and predicted infrared spectra of HeCO2: Dependence on the antisymmetric stretch of CO2, J. Chem. Phys. 128, 124323 (2008).

16 R. Chen, E. Jiao, H. Zhu, and D. Xie, A new ab initio potential energy surface and microwave and infrared spectra for the NeCO2 complex, J. Chem. Phys. 133, 104302 (2010).

17 R. Chen, H. Zhu, and D. Xie, Intermolecular potential energy surface, microwave and infrared spectra of the KrCO2 complex from ab initio calculations, Chem. Phys. Lett. 511, 229 - 234 (2011).

$18 \mathrm{H}$. Zhu, Y. Zhou, and D. Xie, Ab initio intermolecular potential-energy surface and microwave spectra for the NeOCS complex, J. Chem. Phys. 122, 234312 (2005).

19 H. Zhu, Y. Guo, Y. Xue, and D. Xie, Ab initio potential energy surface and predicted microwave spectra for Ar-OCS dimer and structures of Arn-OCS $(\mathrm{n}=214)$ clusters, J. Comput. Chem. 27, 1045-1053 (2006).

${ }^{20}$ E. Feng, C. Sun, C. Yu, X. Shao, and W. Huang, Ab initio potential energy surface and bound states for the KrOCS complex, J. Chem. Phys. 135, 124301 (2011).

21 R. R. Toczyłowski, F. Doloresco, and S. M. Cybulski, Theoretical study of the HeHCN, NeHCN, ArHCN, and KrHCN complexes, J. Chem. Phys. 114, 851-864 (2001).

22 C. R. Munteanu and B. Fernández, Accurate intermolecular ground-state potential-energy surfaces of the HCCHHe, Ne, and Ar van der Waals complexes, J. Chem. Phys. 123, 014309 (2005).

${ }^{23}$ C. Lauzin, E. Cauët, J. Demaison, M. Herman, H. Stoll, and J. Liévin, Accurate ground-state potential energy surfaces of the C2H2Kr and C2H2Xe van der Waals complexes, Mol. Phys. 110, 2751-2760 (2012). 